\title{
Monotone Comparative Statics for Games With Strategic Substitutes*
}

\author{
Sunanda Roy \\ Department of Economics \\ Iowa State University \\ Ames IA, 50011, USA \\ sunanda@iastate.edu
}

\author{
Tarun Sabarwal \\ Department of Economics \\ University of Kansas \\ Lawrence KS, 66045, USA \\ sabarwal@ku.edu
}

\begin{abstract}
Under some conditions, parameterized games with strategic substitutes exhibit monotone comparative statics of equilibria. These conditions relate to a tradeoff between a direct parameter effect and an opposing, indirect strategic substitute effect. If the indirect effect does not dominate the direct effect, monotone comparative statics of equilibria are guaranteed. These conditions are available for best-response functions, differentiable payoff functions, and general payoff functions. Results are extended to correspondences, the analysis applies to asymmetric equilibria, and several examples are provided.
\end{abstract}

JEL Numbers: C70, C72, C62

Keywords: Monotone comparative statics, Nonincreasing functions, Strategic substitutes, Parameterized games

First Draft: November 2004

This Version: May 25, 2010

\footnotetext{
*For helpful comments, we are grateful to Rabah Amir, Federico Echenique, Martin Jensen, Charles Moul, John Nachbar, Wilhelm Neuefeind, Kevin Reffet, Koji Shirai, anonymous referees, and seminar audiences at Drake University, Iowa State University, Queen's University, University of Kansas, Washington University in Saint Louis, Midwest Economic Theory, Kansas Workshop in Economic Theory, Sorbonne Workshop in Economic Theory, SAET 2009, GETA 2009, FESAMES 2009, and SEA 2009. Sabarwal acknowledges financial support from the University of Kansas.
} 


\section{Introduction}

Games with strategic substitutes (GSS) and games with strategic complements (GSC) formalize two basic economic interactions 1 In GSS, if one player takes a higher (or more aggressive, or more intensive) action, a given player's best-response is to take a lower action. That is, best-response of each player is weakly decreasing in actions of the other players. For example, consider a simple, textbook Cournot duopoly, where a given firm's profit-maximizing output is lower when the other firm increases its output. In GSC, if one player takes a higher action, a given player's best response is to take a higher action, too. That is, best-response of each player is weakly increasing in actions of the other players. For example, consider a game of network externalities, where a given player's marginal benefit from adopting a technology is increasing as more other players adopt the same technology.

Monotone comparative statics arise in many applications. The main question is: in a parameterized game, if a parameter increases, when will equilibrium actions of all players increase?

For parameterized GSC, the problem of monotone comparative statics is well-understood 2 but for parameterized GSS, general results are less commonly available. In this paper, we provide some results in this direction.

Notice that there does not appear to be a general result that can be applied to show increasing equilibria in the following simple, asymmetric Cournot duopoly. Consider a linear inverse market demand curve given by $p=a-b\left(x_{1}+x_{2}\right)$, where $x_{1}$ is output of firm 1 , and $x_{2}$ of firm 2. Suppose each firm has constant marginal cost $c$. Moreover, there is a parameterized subsidy of $t \leq c$ per unit, split with an exogenously specified share $\frac{3}{5}$ for firm 1 and $\frac{2}{5}$ for firm 23 Thus, firm 1's marginal cost net of subsidy is $c-\frac{3}{5} t$, and that of firm 2 is $c-\frac{2}{5} t$. In this case, the unique equilibrium is given by $x^{*}(t) \equiv\left(x_{1}^{*}(t), x_{2}^{*}(t)\right)=\left(\frac{a-c+\left(\frac{9}{5}-1\right) t}{3 b}, \frac{a-c+\left(2-\frac{9}{5}\right) t}{3 b}\right)$.

With the standard product order on strategy spaces, this example does not fit the framework of Milgrom and Shannon (1994), because the profit functions are not quasisupermodular 4 Therefore, this game is not supermodular, and this example does not fit the framework of Topkis (1979), Sobel (1988), Vives (1990), or Milgrom and Roberts (1990).

\footnotetext{
${ }^{1}$ Such games are defined in Bulow, Geanakoplos, and Klemperer (1985), and as they show, models of strategic investment, entry deterrence, technological innovation, dumping in international trade, natural resource extraction, business portfolio selection, and others can be viewed in a more unifying framework according as the variables under consideration are strategic complements or strategic substitutes. Earlier developments are provided in Topkis (1978) and Topkis (1979).

${ }^{2}$ Some of this work can be seen in Topkis (1979), Lippman, Mamer, and McCardle (1987), Sobel (1988), Milgrom and Roberts (1990), Vives (1990), Zhou (1994), Milgrom and Shannon (1994), Milgrom and Roberts (1994), Shannon (1995), Villas-Boas (1997), Edlin and Shannon (1998), Echenique (2002), Echenique and Sabarwal (2003), and Quah (2007) among others. Extensive bibliographies are available in Topkis (1998), in Vives (1999), and in Vives (2005).

${ }^{3}$ Alternatively, the parameter $t$ can be thought of as technological improvement, and $\left(\frac{3}{5}, \frac{2}{5}\right)$ can be thought of as differential adaptation of technological improvement. A slightly more general example is presented later.

${ }^{4}$ Denote profit of firm 1 at $\left(x_{1}, x_{2}, t\right)$ by $f^{1}\left(x_{1}, x_{2}, t\right)$, and consider the values $a=10, b=1, c=1, t=0$, and consider $\left(x_{1}, x_{2}\right)=(3,2)$, and $\left(x_{1}^{\prime}, x_{2}^{\prime}\right)=(4,3)$. Then, $f^{1}\left(x_{1}^{\prime}, x_{2}, t\right) \geq f^{1}\left(x_{1}, x_{2}, t\right)$, but $f^{1}\left(x_{1}^{\prime}, x_{2}^{\prime}, t\right)<$ $f^{1}\left(x_{1}, x_{2}^{\prime}, t\right)$.
} 
If the order on one of the strategy spaces is reversed, then it is known (see, for example, Milgrom and Shannon (1994), and Amir (1996)) that this example is a quasi-supermodular game. Of course, such a transformation can violate the single-crossing property in (player strategy; parameter), and therefore, standard results about monotone comparative statics might still not apply. 5 Moreover, asymmetric Cournot conditions rule out an application of Amir and Lambson (2000), and of the intersection point theorem of Tarski (1955)6

In parameterized GSC, monotone comparative statics are typically shown as follows. First, one shows that the equilibrium set is a complete lattice, and therefore, there exists a smallest and a largest equilibrium. Next, one shows that both the smallest and the largest equilibrium increase when the parameter increases.

Roy and Sabarwal (2008) show what goes wrong when such techniques established for GSC are used to analyze GSS. They show that the equilibrium set in GSS is a complete lattice, if, and only if, the game has a unique equilibrium. Indeed, with multiple equilibria, the equilibrium set is completely unordered; that is, no two equilibria are comparable. In particular, there cannot be a smallest and a largest equilibrium. Therefore, with multiple equilibria, techniques for strategic complements that use the complete lattice structure of the equilibrium set, and especially the existence of a largest and smallest equilibrium, are invalid for GSS.

In this paper, we show that using different techniques, it is possible to recover a version of monotone comparative statics for GSS. Our main insight is to observe that in parameterized GSS, when a parameter increases, there are two opposing effects - a direct effect and an indirect effect. The direct effect increases a given player's best-response, and serves to increase a given player's new equilibrium strategy. The indirect effect works as follows: an increase in the parameter increases the best-response of the competitor players, and with strategic substitutes, this tends to indirectly lower a given player's new equilibrium strategy 7

A key result here is to show that when a parameter increases, if the indirect effect does not dominate the direct effect, then a higher equilibrium is guaranteed. The various conditions presented in this paper can be viewed as different ways to identify measures of this combined effect. The result applies to symmetric and asymmetric equilibria.

This result is extended in several directions. Particularly useful for applications is an extension of this result to $N$-player games in which strategies are real-valued, payoff functions are twice continuously differentiable, and best-responses are singleton-valued. The transparency and ease-of-use of this condition makes it a valuable tool to analyze applications that could not be analyzed easily using earlier results. Applications considered here include Cournot duopoly, tournaments, and common-pool resource games. The result is extended

\footnotetext{
${ }^{5}$ Indeed, as shown below, in slight variations of this game, it is easy to have the equilibrium strategy of either player increasing and that of the other player decreasing.

${ }^{6}$ Tarski's intersection point theorem applies only to linearly ordered spaces, and the trick of composing the best response functions for the special duopoly case may not necessarily work with asymmetric equilibria. Indeed, as shown below, it is easy to formulate examples of simple Cournot duopolies where the equilibrium is increasing for one player, and decreasing for the other. The same point applies to techniques that apply when the best-response of one player depends only on the aggregate best-response of other players.

${ }^{7}$ Notably, in GSC, both effects work in the same direction.
} 
to differentiable payoff functions with multi-dimensional strategies and parameters, and to general payoff functions. Similar results are shown for best-response correspondences.

Recall that as shown by Villas-Boas (1997), in parameterized GSS, equilibria do not decrease when a parameter increases. But in general, this result cannot be strengthened to conclude increasing equilibria. 8

Moreover, as shown by Villas-Boas (1997), in the case of a Cournot oligopoly, if an analyst can choose a new partial order, then under certain conditions, there exists a new partial order in which equilibria are increasing. The new partial order, however, might not necessarily be intuitive or relevant for natural parametric policy experiments. For example, for a Cournot oligopoly, the product order may be natural when considering the impact of taxes or subsidies on firm output, and the existence of some other partial order under which equilibria are increasing might not be interesting. The results here apply to cases where a partial order is considered as fixed.

The paper proceeds as follows. Section 2 presents the main ideas and the results for best response functions and differentiable payoff functions. Section 3 presents results for general payoff functions. Section 4 provides some examples. Finally, section 5 extends the results to correspondences.

\section{Monotone Comparative Statics}

Consider the basic problem of monotone comparative statics: if a parameter increases, when will equilibrium actions of all players increase as well?

This question can alternatively be viewed in the more general context of providing incentives to players to try and internalize an externality. Lets think of players as firms for now, engaged in decentralized Cournot competition, and producing an output with a positive externality, say, wireless internet gateways, and suppose it is regulatory policy to encourage additional internet access, by, say, subsidizing production.

It is well-known that if a particular firm gets a subsidy, its output will go up. But then strategic substitutes imply that the output of the other firms will go down. Moreover, if the other firms receive a subsidy as well, they will show a tendency to increase output. This tendency will serve to depress the output of the first firm, due to the strategic substitute effect. Therefore, it is unclear if a subsidy in the presence of strategic substitutes necessarily increases firm output. Consequently, in oligopoly markets with externalities, it may be hard for regulators to create decentralized incentives to internalize the externalities and increase output for all firms 9

\footnotetext{
${ }^{8} \mathrm{~A}$ more specialized result is available, too. When player strategy spaces are chains, symmetric equilibria are nondecreasing in the parameter. This can be inferred from results in, for example, Milgrom and Roberts (1994), and Villas-Boas (1997). Another derivation can be found in Roy and Sabarwal (2008). Additionally, some aspects of non-monotone mappings that are increasing in some variables and decreasing in others are explored in Roy (2002).

${ }^{9} \mathrm{~A}$ regulator might not care about increasing total output only, because that is consistent with expanding one firm, and contracting the other, which may lead to anticompetitive behavior.
} 
In order to fix ideas, let's consider the following interpretive example that shows how asymmetry can affect monotone comparative statics.

Example 1. (Simple Cournot Duopoly.) Consider a standard Cournot duopoly with a linear inverse market demand curve given by $p=a-b\left(x_{1}+x_{2}\right)$, where $x_{1}$ is output of firm 1, and $x_{2}$ of firm 2. Suppose each firm has constant marginal cost, $c$. Moreover, there is a subsidy of $t \leq c$ per unit, split with share $\xi \in[0,1]$ for firm 1 , and share $1-\xi$ for firm 210 Thus, marginal cost net of subsidy is $c-\xi t$ for firm 1 , and $c-(1-\xi) t$ for firm 2. The best-response function for firm 1 is $g^{1}\left(x_{2}, t\right)=\frac{a-c+\xi t-b x_{2}}{2 b}$, and for firm 2 is $g^{2}\left(x_{1}, t\right)=\frac{a-c+(1-\xi) t-b x_{1}}{2 b}$.

With the standard product order, it is easy to check that the joint best-response function, $g\left(x_{1}, x_{2}, t\right) \equiv\left(g^{1}\left(x_{2}, t\right), g^{2}\left(x_{1}, t\right)\right)$, is strictly decreasing in $\left(x_{1}, x_{2}\right)$, and is strictly increasing in $t$. The unique equilibrium at $t$ is $x^{*}(t) \equiv\left(x_{1}^{*}(t), x_{2}^{*}(t)\right)=\left(\frac{a-c+(3 \xi-1) t}{3 b}, \frac{a-c+(2-3 \xi) t}{3 b}\right)$. Consequently,

$$
\begin{array}{ll}
\xi<\frac{1}{3} & \Leftrightarrow x_{1}^{*}(t) \text { is decreasing in } t \text {, and } x_{2}^{*}(t) \text { is increasing in } t, \\
\frac{1}{3} \leq \xi \leq \frac{2}{3} & \Leftrightarrow x_{1}^{*}(t) \text { is increasing in } t \text {, and } x_{2}^{*}(t) \text { is increasing in } t \text {, and } \\
\frac{2}{3}<\xi & \Leftrightarrow x_{1}^{*}(t) \text { is increasing in } t \text {, and } x_{2}^{*}(t) \text { is decreasing in } t \text {. }
\end{array}
$$

In particular, equilibrium is monotone nondecreasing in $t$, if, and only if, $\frac{1}{3} \leq \xi \leq \frac{2}{3}$. Therefore, this example shows the possibility of monotone comparative statics with asymmetric equilibria $\left(\xi \neq \frac{1}{2}\right)$, as long as the asymmetry is not too large.

More generally, consider a triple $(X, T, g)$, where $(X, \preceq)$ is a nonempty partially ordered set, $T$ is a nonempty partially ordered set 11 and $g: X \times T \rightarrow X$ is a function. For a triple $(X, T, g)$, consider the following assumptions.

Assumption I.A: For every $x \in X, g(x, \cdot)$ is nondecreasing in $t .12$ and for every $t \in T$, $g(\cdot, t)$ is nonincreasing in $x 13$

Assumption I.B: $(X, \preceq)$ is a nonempty, compact, convex, sublattice of a Banach lattice; order intervals in $X$ are closed and convex; and for every $t, g(\cdot, t)$ is continuous.

Assumption I.C: $(X, \preceq)$ is a nonempty, closed, bounded, convex, sublattice of a Banach lattice; order intervals in $X$ are closed and convex; and for every $t, g(\cdot, t)$ is a compact operator.

In a parameterized GSS, each player's best response function is nonincreasing in other player strategies, and therefore, the product of the best-response functions of the players satisfies the nonincreasing-in- $x$ property (in the product order). Similarly, when each player's best-response function is nondecreasing in the parameter $t$, then the product of the bestresponse functions of the players satisfies the nondecreasing-in- $t$ property (in the product order). Of course, as stated, the assumptions do not require that $X$ be a product of individual strategy spaces, or it be endowed with a product order.

\footnotetext{
${ }^{10}$ The example in the introduction is the case where $\xi=\frac{3}{5}$.

${ }^{11}$ Throughout the paper, when no confusion arises, the same symbol $\preceq$ denotes the partial order on $T$.

${ }^{12}$ For every $x$, and for every $t, \hat{t} \in T, t \preceq \hat{t} \Rightarrow g(x, t) \preceq g(x, \hat{t})$.

${ }^{13}$ For every $t$, and for every $x, y \in X, x \preceq y \Rightarrow g(y, t) \preceq g(x, t)$.
} 
A triple $(X, T, g)$ is admissible if it satisfies either I.A and I.B, or I.A and I.C 14 For each $t$, let $\mathcal{E}(t)=\{x \in X \mid x=g(x, t)\}$ be the equilibrium (or fixed) points of $g$ at $t$. Schauder's theorem implies that for every $t, \mathcal{E}(t)$ is non-empty. Here is a key result.

Theorem 1. Let $(X, T, g)$ be an admissible triple. Fix $t^{*} \in T$, and let $x^{*} \in \mathcal{E}\left(t^{*}\right)$. Consider $\hat{t} \in T$ such that $t^{*} \preceq \hat{t}$, and let $\hat{y}=g\left(x^{*}, \hat{t}\right)$, and $\hat{x}=g(\hat{y}, \hat{t})$.

If $x^{*} \preceq \hat{x}$, then there is $\hat{x}^{*} \in \mathcal{E}(\hat{t})$ such that $x^{*} \preceq \hat{x}^{*}$.

Proof. Notice that $x^{*} \preceq \hat{y}$, because $g$ is nondecreasing in $t$. Moreover, for every $x$ in $\left[x^{*}, \hat{y}\right], g(x, \hat{t}) \in\left[x^{*}, \hat{y}\right]$, and this can be seen as follows. Suppose $x^{*} \preceq x \preceq \hat{y}$. Then $x \preceq \hat{y}$ implies that $g(x, \hat{t}) \succeq g(\hat{y}, \hat{t}) \succeq x^{*}$, where the first inequality follows from the fact that $g(\cdot, \hat{t})$ is nonincreasing, and the second follows from the condition in the theorem. Moreover, $x^{*} \preceq x$ implies that $g(x, \hat{t}) \preceq g\left(x^{*}, \hat{t}\right)=\hat{y}$, where the inequality follows from nonincreasing $g(\cdot, \hat{t})$, and the equality follows from definition of $\hat{y}$. Therefore, the restriction of $g(\cdot, \hat{t})$ to $\left[x^{*}, \hat{y}\right]$ is a map from $\left[x^{*}, \hat{y}\right]$ to $\left[x^{*}, \hat{y}\right]$. By Schauder's theorem, there is $\hat{x}^{*} \in\left[x^{*}, \hat{y}\right]$ such that $g\left(\hat{x}^{*}, \hat{t}\right)=\hat{x}^{*}$, and consequently, there is $\hat{x}^{*} \in \mathcal{E}(\hat{t})$ such that $x^{*} \preceq \hat{x}^{*}$.

As mentioned above, this theorem does not require that $X$ be a product of individual strategy spaces, or it be endowed with a product order.

Moreover, the fact that order intervals are compact and convex is used only to guarantee existence of an equilibrium. In classes of games where an equilibrium always exists, these assumptions are not needed to prove theorem 1. For example, in quasi-aggregative games, see Jensen (2010), equilibrium existence is guaranteed without convexity or quasi-concavity assumptions, and therefore, our proof will work by invoking equilibrium existence on $\left[x^{*}, \hat{y}\right]$, and not requiring convexity or quasi-concavity.

The intuition behind the condition in this theorem can be seen clearly in a two-player game, with players indexed $i=1,2$. Suppose player $i$ 's strategies lie in a non-empty, compact, convex interval $X^{i}$ of the real numbers, and there is a partially ordered parameter space $T$. Player $i$ 's best-response function is $g^{i}: X^{j} \times T \rightarrow X^{i}$, with $i \neq j$. For each $i$ and $t$, suppose $g^{i}(\cdot, t)$ is nonincreasing, and for each $i$, and for each $x_{j} \in X^{j}$, suppose $g^{i}\left(x_{j}, \cdot\right)$ is nondecreasing. Let $X=X^{1} \times X^{2}$, endowed with the product order (denoted $\preceq$ ). Suppose $g\left(x_{1}, x_{2}, t\right) \equiv\left(g^{1}\left(x_{2}, t\right), g^{2}\left(x_{1}, t\right)\right)$ is continuous in $\left(x_{1}, x_{2}\right)$. Theorem 1 implies:

Corollary 1. Consider a two-player game, as above. Fix $t^{*}, \hat{t} \in T$, with $t^{*} \preceq \hat{t}$, and let $x^{*}=\left(x_{1}^{*}, x_{2}^{*}\right) \in \mathcal{E}\left(t^{*}\right)$. Let $\left(\hat{y}_{1}, \hat{y}_{2}\right)=\left(g^{1}\left(x_{2}^{*}, \hat{t}\right), g^{2}\left(x_{1}^{*}, \hat{t}\right)\right)$, and $\left(\hat{x}_{1}, \hat{x}_{2}\right)=\left(g^{1}\left(\hat{y}_{2}, \hat{t}\right), g^{2}\left(\hat{y}_{1}, \hat{t}\right)\right)$. If $x_{1}^{*} \leq \hat{x}_{1}$ and $x_{2}^{*} \leq \hat{x}_{2}$, then there is $\hat{x}^{*}=\left(\hat{x}_{1}^{*}, \hat{x}_{2}^{*}\right) \in \mathcal{E}(\hat{t})$ such that $x^{*} \preceq \hat{x}^{*}$.

The conditions in this corollary can be viewed as follows. Starting from an existing equilibrium, $x^{*}=\left(x_{1}^{*}, x_{2}^{*}\right)$ at $t=t^{*}$, an increase in $t$ has two effects on $g^{1}(\cdot, \cdot)$. One effect is an increase in $g^{1}$, because best-response functions are nondecreasing in $t$. (This is a direct

\footnotetext{
${ }^{14}$ Notice that assumption I.A is an integral component of a parameterized GSS, whereas assumption I.B or I.C is made to guarantee existence of an equilibrium via Brouwer-Schauder type theorems. In particular, in I.B or I.C, the assumption on order intervals in $X$ is automatically satisfied in standard Banach lattices such as $\mathbb{R}^{n}, L_{p}(\mu)$ spaces, space of continuous functions over a compact set, and so on. In I.A, I.B, and I.C the partial order on $X$ is assumed to be the same, and the order and the topological structure on $X$ are assumed to be compatible in terms of lattice norms. See, for example, Aliprantis and Border (1994).
} 


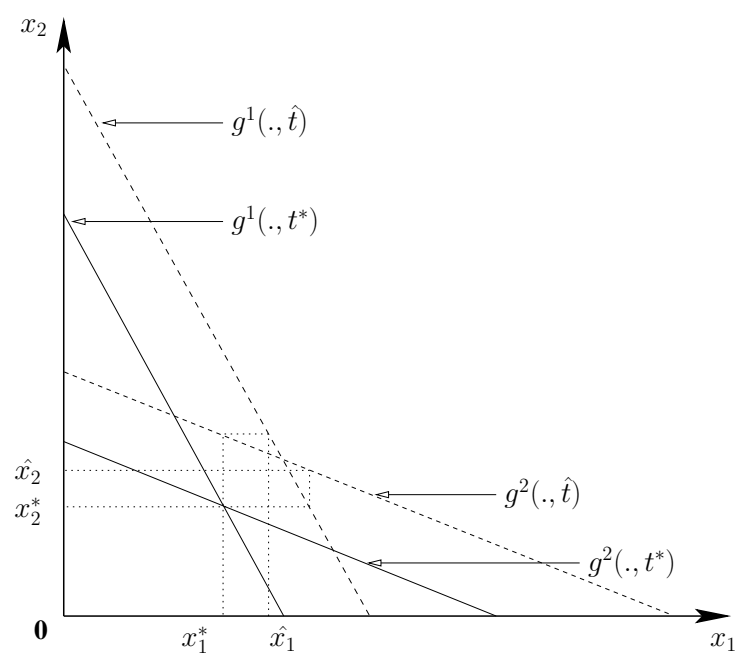

Figure 1: Existence of Increasing Equilibria

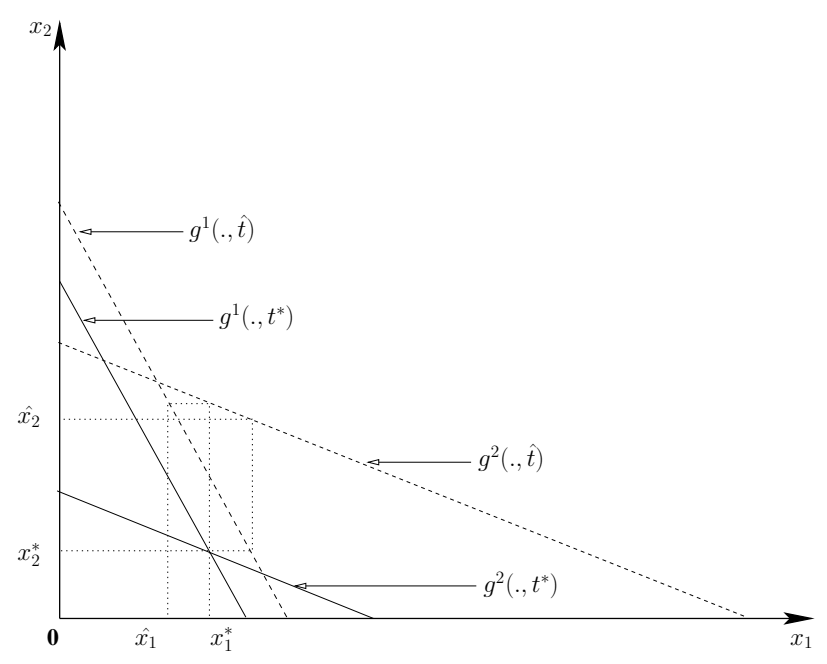

Figure 2: Violation of first condition

effect of an increase in $t$.) The other effect is a decrease in $g^{1}$, because an increase in $t$ increases $g^{2}\left(x_{2}^{*}, t\right)$, and $x_{1}$ and $x_{2}$ are strategic substitutes. (This is an indirect effect arising from player 1's response to player 2's response to an increase in $t$.) Similar statements are valid for player 2 as well. Taken together, the conditions say that for each player, as long as the indirect strategic substitute effect does not dominate the direct parameter effect, there is a new equilibrium that is larger than $x^{*}=\left(x_{1}^{*}, x_{2}^{*}\right)$. The intuition for the general case is similar. A simple graphical illustration of these conditions is presented in Figure 1.

It is useful to note that if either condition in the corollary is not satisfied, this result may not necessarily apply. This can be seen graphically in figure 2 , where the first condition is violated but the second condition is satisfied. An alternative figure can be constructed similarly where the reverse is true.

The condition in theorem 1 can be translated to payoff functions by noticing that if $x^{*}$ is an equilibrium at $t^{*}$, and if $g\left(g\left(x^{*}, t\right), t\right)$ is increasing in $t$ at $t^{*}$, then the condition in theorem 1 is satisfied for an increase in $t$ from $t^{*}$. For twice continuously differentiable payoff functions, this insight leads to the following transparent, and easy-to-use condition.

Suppose there are $N$ players, strategies and parameters are real-valued, and payoff function of player $i$ is given by $f^{i}\left(x_{i}, x_{-i}, t\right)$, where for each $\left(x_{-i}, t\right)$, $\arg \max _{x_{i} \in X^{i}} f^{i}\left(x_{i}, x_{-i}, t\right)$ is singleton-valued 15 and for each $i, f^{i}$ is $C^{2}$. In this case, for player $i=1$, the condition in the theorem 1 is satisfied, if

$$
\left.\frac{\partial}{\partial t}\left(g^{1}\left(g^{2}\left(x_{-2}, t\right), g^{3}\left(x_{-3}, t\right), \ldots, g^{N}\left(x_{-N}, t\right), t\right)\right)\right|_{\left(x^{*}, t^{*}\right)}>0
$$

and for each player $i=2, \ldots, N$, a similar condition with an appropriate change of index 16

\footnotetext{
${ }^{15}$ This is true when $f^{i}$ is strictly quasi-concave in $x_{i}$, or strictly concave in $x_{i}$.

${ }^{16}$ As usual, to apply this version, we suppose that the derivative is well-defined; in particular, $\left(x^{*}, t^{*}\right)$ is in the interior.
} 
Using the Implicit Function theorem, it is easy to calculate that

$$
\left.\frac{\partial}{\partial t}\left(g^{1}\left(g^{2}\left(x_{-2}, t\right), \ldots, g^{N}\left(x_{-N}, t\right), t\right)\right)\right|_{\left(x^{*}, t^{*}\right)}>0 \Leftrightarrow f_{1, t}^{1}+\left.\sum_{n=2}^{N} f_{1, n}^{1}\left(-\frac{f_{n, t}^{n}}{f_{n, n}^{n}}\right)\right|_{\left(x^{*}, t^{*}\right)}>0
$$

where a superscript on a payoff function $f$ indexes a player, and subscripts denote partial derivatives. Thus, for example, $f_{i, n}^{i}=\frac{\partial^{2} f^{i}}{\partial x_{i} \partial x_{n}}$, and $f_{i, t}^{i}=\frac{\partial^{2} f^{i}}{\partial x_{i} \partial t} 17$

With an appropriate change of index, it follows that if for every $i=1, \ldots, N$,

$$
f_{i, t}^{i}+\left.\sum_{n \neq i} f_{i, n}^{i}\left(-\frac{f_{n, t}^{n}}{f_{n, n}^{n}}\right)\right|_{\left(x^{*}, t^{*}\right)}>0
$$

then for small increases in $t$ from $t^{*}$, the condition in theorem 1 is satisfied.

These conditions have the same intuition as earlier. For a given player $i, f_{i, t}^{i}$ is positive, and captures the direct effect of an increase in $t$. The term $\sum_{n \neq i} f_{i, n}^{i}\left(-\frac{f_{n, t}^{n}}{f_{n, n}^{n}}\right)$ is negative, and captures the indirect effect of an increase in $t$, one for each competitor. As above, if the indirect effect does not dominate the direct effect, monotone comparative statics are guaranteed. Notice that in this use of the Implicit Function theorem, we only require knowledge of how best-responses are behaving around $t^{*}$, not how fixed points of best-responses are behaving around $t^{*}$. This yields two benefits: it is computationally easier, and it provides economically intuitive conditions. These benefits are typically absent in standard applications of the Implicit Function theorem to the equilibrium set.

Similarly, if for each player $i=1, \ldots, N$, $i$ 's strategy space is multi-dimensional, say $X^{i} \subset \mathbb{R}^{N_{i}}$, and the parameter space is multi-dimensional, say, $T \subset \mathbb{R}^{N_{0}}$. Then again, for player $i=1$, the relevant condition is

$$
\begin{aligned}
&\left.D_{t}\left(g^{1}\left(g^{2}\left(x_{-2}, t\right), \ldots, g^{N}\left(x_{-N}, t\right), t\right)\right)\right|_{\left(x^{*}, t^{*}\right)} \\
& \quad=-\left.\left[D_{1} f_{1}^{1}\right]^{-1}\left[D_{t} f_{1}^{1}+\sum_{n=2}^{N} D_{n} f_{1}^{1}\left(-\left[D_{n} f_{n}^{n}\right]^{-1} D_{t} f_{n}^{n}\right)\right]\right|_{\left(x^{*}, t^{*}\right)} \geq 0 .
\end{aligned}
$$

For reference, notice that the dimension of the matrix on either side of the inequality is $N_{1} \times N_{0}$. When $N_{0}=1$, the above condition is a vector inequality, and when $N_{0}>1$, the linear operator in the above condition is a positive operator. If a similar condition holds for each $i$, then the condition in theorem 1 is satisfied. The following corollary to theorem 1 is useful to exhibit increasing selections of equilibria.

Corollary 2. Let $(X, T, g)$ be an admissible triple. If for every $x, g(g(x, t), t)$ is nondecreasing in $t$, then for every $t^{*} \preceq \hat{t}$, and for every $x^{*} \in \mathcal{E}\left(t^{*}\right)$, there is $\hat{x}^{*} \in \mathcal{E}(\hat{t})$ such that $x^{*} \preceq \hat{x}^{*}$.

\footnotetext{
${ }^{17}$ Notice that in a parameterized game of strategic substitutes, for $n \neq i, f_{i, n}^{i}<0$ formalizes strategic substitutes, and for $n=i, f_{i, n}^{i}<0$ formalizes strict concavity in own argument. Moreover, $f_{i, t}^{i}>0$ formalizes increasing differences in $t$.
} 
The following corollary presents a version of strong monotone comparative statics; that is, all equilibria at $\hat{t}$ are greater than $x^{*}$.

Corollary 3. Let $(X, T, g)$ be an admissible triple, with $X$ a complete lattice. Fix $t^{*} \preceq \hat{t}$, and let $x^{*} \in \mathcal{E}\left(t^{*}\right)$. Consider $g(g(x, \hat{t}), \hat{t})$, and let $\hat{x}_{L}=i n f_{X}\{x \mid g(g(x, \hat{t}), \hat{t}) \preceq x\}$.

If $x^{*} \preceq \hat{x}_{L}$, then for every $\hat{x}^{*} \in \mathcal{E}(\hat{t}), \quad x^{*} \preceq \hat{x}^{*}$, and

If $x^{*} \prec \hat{x}_{L}$, then for every $\hat{x}^{*} \in \mathcal{E}(\hat{t}), \quad x^{*} \prec \hat{x}^{*}$.

Proof. Notice that $g(g(x, \hat{t}), \hat{t})$, is nondecreasing in $x$, and therefore, by Tarski's theorem, $\hat{x}_{L}$ exists, and is the smallest fixed point of $g(g(x, \hat{t}), \hat{t})$ at $\hat{t}$. Moreover, the set of fixed points of $g(g(x, \hat{t}), \hat{t})$ at $\hat{t}$ is a complete lattice. The result now follows by noting that the set of fixed points of $g$ at $\hat{t}$ is a subset of the set of fixed points of $g(g(x, \hat{t}), \hat{t})$ at $\hat{t}$.

Another condition that guarantees the conclusion of the second statement (strictly increasing equilibria) in this corollary is the following.

$$
\text { If } x^{*} \preceq \hat{x}_{L} \text {, and if } \mathcal{E}(\hat{t}) \text { is not a singleton, then for every } \hat{x}^{*} \in \mathcal{E}(\hat{t}), x^{*} \prec \hat{x}^{*} \text {. }
$$

This statement can be proved using results in Dacic (1981), and Roy and Sabarwal (2008), which imply that in games with strategic substitutes, the equilibrium set is completely unordered; that is, no two elements are comparable. Therefore, if $x^{*} \preceq \hat{x}_{L}$, and if $\mathcal{E}(\hat{t})$ is not a singleton, then $x^{*} \notin \mathcal{E}(\hat{t})$, and the result follows.

\section{Conditions on Payoff Functions}

This section defines general parameterized GSS, and provides conditions on payoff functions that yield the analogue of theorem 1 above. In order to do so, it is helpful to understand first conditions on payoff functions that guarantee nonincreasing best-responses. These are presented in the next subsection.

\subsection{A Monotonicity Theorem}

Recall from Milgrom and Shannon (1994) that when $X$ is a lattice 18 a function $f: X \rightarrow \mathbb{R}$ is quasi-supermodular if (1) $f(x) \geq f(x \wedge y) \Longrightarrow f(x \vee y) \geq f(y)$, and $(2) f(x)>$ $f(x \wedge y) \Longrightarrow f(x \vee y)>f(y)$. Moreover, when $X$ is a lattice and $T$ is a partially ordered set, a function $f: X \times T \rightarrow \mathbb{R}$ satisfies single-crossing property in $(x ; t)$, if for every $x^{\prime} \succ x^{\prime \prime}$ and $t^{\prime} \succ t^{\prime \prime},(1) f\left(x^{\prime}, t^{\prime \prime}\right)>f\left(x^{\prime \prime}, t^{\prime \prime}\right) \Longrightarrow f\left(x^{\prime}, t^{\prime}\right)>f\left(x^{\prime \prime}, t^{\prime}\right)$, and $(2) f\left(x^{\prime}, t^{\prime \prime}\right) \geq f\left(x^{\prime \prime}, t^{\prime \prime}\right) \Longrightarrow$ $f\left(x^{\prime}, t^{\prime}\right) \geq f\left(x^{\prime \prime}, t^{\prime}\right)$. Recall that the single-crossing property is an ordinal condition. Its cardinal version is increasing differences, with the well-known intuition that the function $f\left(x^{\prime}, t\right)-f\left(x^{\prime \prime}, t\right)$ as a function of $t$ crosses zero at most once, and only from below.

We shall say that a function $f: X \times T \rightarrow \mathbb{R}$ satisfies decreasing single-crossing property in $(x ; t)$ if for every $x^{\prime} \succ x^{\prime \prime}$ and $t^{\prime} \succ t^{\prime \prime}$, (1) $f\left(x^{\prime}, t^{\prime \prime}\right) \leq f\left(x^{\prime \prime}, t^{\prime \prime}\right) \Longrightarrow f\left(x^{\prime}, t^{\prime}\right) \leq$

\footnotetext{
${ }^{18}$ This paper uses standard lattice terminology. See, for example, Topkis (1998).
} 
$f\left(x^{\prime \prime}, t^{\prime}\right)$, and $(2) f\left(x^{\prime}, t^{\prime \prime}\right)<f\left(x^{\prime \prime}, t^{\prime \prime}\right) \Longrightarrow f\left(x^{\prime}, t^{\prime}\right)<f\left(x^{\prime \prime}, t^{\prime}\right)$. Analogous to the singlecrossing property, the decreasing single-crossing property is an ordinal condition, and its cardinal version is decreasing differences, which implies that the function $f\left(x^{\prime}, t\right)-f\left(x^{\prime \prime}, t\right)$ as a function of $t$ crosses zero at most once, and only from above. This intuition is the motivation for our present terminology 19

For an order on nonempty subsets of $X$, we use the standard (induced) set order used in the literature. That is, for non-empty subsets $A, B$ of $X, A \sqsubseteq B$ if for every $a \in A$, and for every $b \in B, a \wedge b \in A$, and $a \vee b \in B$, where the operations $\wedge, \vee$ are with respect to $\preceq$.

With these concepts, the proof of the monotonicity theorem in Milgrom and Shannon (1994) can be adapted to prove the following theorem.

Theorem 2. Let $X$ be a lattice, $T$ be a partially ordered set, $S$ be a nonempty subset of $X$, and $f: X \times T \rightarrow \mathbb{R}$. Reverse the standard set order on $X 20$ The following is true.

$\arg \max _{x \in S} f(x, t)$ is monotone nonincreasing in $(t, S)$, if, and only if, $f$ is quasi-supermodular in $x$ and satisfies decreasing single-crossing property in $(x ; t)$

Proof. For each $(t, S)$, let $M(t, S)=\arg \max _{x \in S} f(x, t)$.

$(\Longleftarrow)$ Let $t \preceq t^{\prime}, S^{\prime} \sqsubseteq S, x \in M(t, S), x^{\prime} \in M\left(t^{\prime}, S^{\prime}\right)$. Consider $x \wedge x^{\prime}$. As $x \in$ $M(t, S), f(x, t) \geq f\left(x \vee x^{\prime}, t\right)$. Therefore,

$$
\begin{aligned}
f\left(x \vee x^{\prime}, t\right) \ngtr f(x, t) & \Longrightarrow f\left(x^{\prime}, t\right) \ngtr f\left(x \wedge x^{\prime}, t\right) \\
& \Longleftrightarrow f\left(x^{\prime}, t\right) \leq f\left(x \wedge x^{\prime}, t\right) \\
& \Longrightarrow f\left(x^{\prime}, t^{\prime}\right) \leq f\left(x \wedge x^{\prime}, t^{\prime}\right),
\end{aligned}
$$

where the first implication follow from quasi-supermodularity, and the last implication follows from decreasing single-crossing property. Thus, $x \wedge x^{\prime} \in M\left(t^{\prime}, S^{\prime}\right)$. Similarly, it can be shown that $x \vee x^{\prime} \in M(t, S)$.

$(\Longrightarrow)$ To show that $f$ is quasi-supermodular in $x$, fix $t \in T$, and $x, x^{\prime} \in X$. Let $S=$ $\left\{x, x \vee x^{\prime}\right\}$, and $S^{\prime}=\left\{x^{\prime}, x \wedge x^{\prime}\right\}$. Then $S^{\prime} \sqsubseteq S$. Suppose $f\left(x \vee x^{\prime}, t\right) \leq f(x, t)$. Then $x \in M(t, S)$. But then, as $M(\cdot, \cdot)$ is nonincreasing, it follows that $x \wedge x^{\prime} \in M\left(t, S^{\prime}\right)$, whence $f\left(x^{\prime}, t\right) \leq f\left(x \wedge x^{\prime}, t\right)$. The proof for strict inequality is similar.

To show that $f$ satisfies decreasing single-crossing property in $(x ; t)$, fix $x \prec x^{\prime}$ and $t \prec t^{\prime}$. Let $S=\left\{x, x^{\prime}\right\}$. Suppose $f\left(x^{\prime}, t\right) \leq f(x, t)$. Then $x \in M(t, S)$, and as $M$ is nonincreasing, $x=x \wedge x^{\prime} \in M\left(t^{\prime}, S\right)$. Consequently, $f\left(x^{\prime}, t^{\prime}\right) \leq f\left(x, t^{\prime}\right)$. The proof for strict inequality is similar.

This theorem is interesting in its own right as characterizing nonincreasing solutions in a class of maximization problems. Its main application to parameterized GSS here is the "if" direction, with a player's strategy space given by $X$, with $S$ identically equal to $X$, and with strategy space of other players given by $T$.

\footnotetext{
${ }^{19}$ Amir (1996) proposes a similar "dual single-crossing" property for one-dimensional strategies.

${ }^{20} S$ is lower than $S^{\prime}$ in the reverse order means that $S^{\prime} \sqsubseteq S$.
} 


\subsection{Parameterized Games with Strategic Substitutes}

Consider a set of players $I$, with players indexed by $i \in I$. Each player $i$ has a partially ordered strategy space $\left(X^{i}, \preceq^{i}\right)$. The overall strategy space is the product of $X^{i}$, denoted $X$, and endowed with the product order (and topology). Let $T$ be a partially ordered set of parameters. Each player $i$ has a payoff function, $f^{i}: X \times T \rightarrow \mathbb{R}$, denoted $f^{i}\left(x_{i}, x_{-i}, t\right)$. The collection $\Gamma=\left(I, T,\left(X^{i}, \preceq^{i}, f^{i}\right)_{i \in I}\right)$ is a parameterized game with strategic substitutes, if for every player $i$,

(1) $\left(X^{i}, \preceq^{i}\right)$ is a nonempty, sub-complete, convex, sub-lattice of a Banach lattice, with closed, convex intervals 21 and $f^{i}$ is continuous,

(2) For every $\left(x_{-i}, t\right), f^{i}$ is quasi-supermodular in $x_{i}$,

(3) For every $x_{-i}, f^{i}$ satisfies single-crossing property in $\left(x_{i} ; t\right)$, and

(4) For every $t, f^{i}$ satisfies decreasing single-crossing property in $\left(x_{i} ; x_{-i}\right)$.

Recall that (2) and (3) are standard ingredients of parameterized supermodular and quasi-supermodular games. Single-crossing property in $\left(x_{i} ; t\right)$ allows best responses to be nondecreasing in the parameter. Strategic substitutes are modeled by the assumption of decreasing single-crossing property in $\left(x_{i} ; x_{-i}\right)$. Theorem 2 implies that each player's best response is nonincreasing in other player strategies.

Suppose for each $i$, and for each $\left(x_{-i}, t\right), g^{i}\left(x_{-i}, t\right)=\arg \max _{x_{i} \in S} f^{i}\left(x_{i}, x_{-i}, t\right)$ is singletonvalued, and let $g=\left(g^{i}\right)_{i \in I}$.

Let $t^{*}, \hat{t} \in T$, and $x^{*} \in \mathcal{E}\left(t^{*}\right)$. For player $i, f^{i}$ satisfies monotone comparative statics (MCS) at $\left(x^{*}, t^{*}, \hat{t}\right)$, if for every $x_{i} \succeq x_{i}^{\prime},(1) f^{i}\left(x_{i}, x_{-i}^{*}, t^{*}\right) \geq f^{i}\left(x_{i}^{\prime}, x_{-i}^{*}, t^{*}\right) \Rightarrow f^{i}\left(x_{i}, \hat{y}_{-i}, \hat{t}\right) \geq$ $f^{i}\left(x_{i}^{\prime}, \hat{y}_{-i}, \hat{t}\right)$, and $(2) f^{i}\left(x_{i}, x_{-i}^{*}, t^{*}\right)>f^{i}\left(x_{i}^{\prime}, x_{-i}^{*}, t^{*}\right) \Rightarrow f^{i}\left(x_{i}, \hat{y}_{-i}, \hat{t}\right)>f^{i}\left(x_{i}^{\prime}, \hat{y}_{-i}, \hat{t}\right)$, where $\hat{y}_{-i}=\left(\hat{y}_{j}\right)_{j \neq i}$, and for each $j \neq i, \hat{y}_{j}=\arg \max _{x_{j}} f^{j}\left(x_{j}, x_{-j}^{*}, \hat{t}\right)$ As discussed in detail above, $\hat{y}_{-i}$ is the indirect strategic substitute effect of a change in the parameter from $t^{*}$ to $\hat{t}$, and it determines how much the direct effect has to change to compensate for this opposing indirect effect. The lemma below shows that the combined effect is favorable, if MCS holds.

Lemma 1. If $f^{i}$ satisfies MCS at $\left(x^{*}, t^{*}, \hat{t}\right)$, then $x_{i}^{*} \preceq g^{i}\left(\hat{y}_{-i}, \hat{t}\right)$.

Proof. Let $x_{i}^{*}=g^{i}\left(x_{-i}^{*}, t^{*}\right)$ and $\hat{x}_{i}=g^{i}\left(\hat{y}_{-i}, \hat{t}\right)$. Notice that $f^{i}\left(x_{i}^{*}, x_{-i}^{*}, t^{*}\right) \geq f^{i}\left(x_{i}^{*} \wedge\right.$ $\left.\hat{x}_{i}, x_{-i}^{*}, t^{*}\right)$, and therefore, using quasi-supermodularity, $f^{i}\left(x_{i}^{*} \vee \hat{x}_{i}, x_{-i}^{*}, t^{*}\right) \geq f^{i}\left(\hat{x}_{i}, x_{-i}^{*}, t^{*}\right)$, and therefore, using MCS at $\left(x^{*}, t^{*}, \hat{t}\right), f^{i}\left(x_{i}^{*} \vee \hat{x}_{i}, \hat{y}_{-i}, \hat{t}\right) \geq f^{i}\left(\hat{x}_{i}, \hat{y}_{-i}, \hat{t}\right)$, whence $x_{i}^{*} \vee \hat{x}_{i}=\hat{x}_{i}$. Similarly, $x_{i}^{*} \wedge \hat{x}_{i}=x_{i}^{*}$, and consequently, $x_{i}^{*} \preceq g^{i}\left(\hat{y}_{-i}, \hat{t}\right)$.

Notice that a limitation of the MCS condition is that it requires knowledge of $\hat{y}_{-i}$; that is, how do opponents of $i$ respond to the old equilibrium at the new parameter value. Nevertheless, this condition can be used in examples, as shown in the next section. An alternative condition can be postulated, requiring only an upper bound for the best-response, as follows 23 In the MCS condition, replace each $\hat{y}_{j}$ by a $\tilde{y}_{j}$, where $\tilde{y}_{j} \succeq \arg \max _{x_{j}} f^{j}\left(x_{j}, x_{-j}^{*}, \hat{t}\right)$,

\footnotetext{
${ }^{21}$ As earlier, the order and topological structure are assumed to be compatible in terms of lattice norms.

${ }^{22}$ Notice that this has the flavor of a single crossing property. Indeed, if we let $\hat{y}_{-i}(t)=g_{-i}\left(x^{*}, t\right)$, and consider the single-crossing property in $\left(x_{i} ; t\right)$ for the function $\left(x_{i}, t\right) \mapsto f^{i}\left(x_{i}, \hat{y}_{-i}(t), t\right)$, then the above is a restricted version of that property.

${ }^{23} \mathrm{We}$ are grateful to an anonymous referee for pointing out this condition.
} 
and replace each $\hat{y}_{-i}$ by $\tilde{y}_{-i}$. Using $\hat{y}_{j}=\arg \max _{x_{j}} f^{j}\left(x_{j}, x_{-j}^{*}, \hat{t}\right)$, and $\hat{y}_{-i}=\left(\hat{y}_{j}\right)_{j \neq i}$, lemma 1 remains true as stated. In its proof, replace $\hat{y}_{-i}$ by $\tilde{y}_{-i}$ everywhere, to get $x_{i}^{*} \preceq g^{i}\left(\tilde{y}_{-i}, \hat{t}\right)$. Now, $\hat{y}_{-i} \preceq \tilde{y}_{-i}$ and $g^{i}$ is nonincreasing in $y_{-i}$ imply $x_{i}^{*} \preceq g^{i}\left(\hat{y}_{-i}, \hat{t}\right)$, as desired.

Let $\Gamma$ be a parameterized GSS, $t^{*} \in T$, and $x^{*} \in \mathcal{E}\left(t^{*}\right)$. $\Gamma$ satisfies $\boldsymbol{M C S}$ at $\left(x^{*}, t^{*}\right)$, if there is $\hat{t} \succ t^{*}$ such that for all $i, f^{i}$ satisfies MCS at $\left(x^{*}, t^{*}, \hat{t}\right)$. $\Gamma$ satisfies all parameter $\boldsymbol{M C S} \boldsymbol{a t}\left(x^{*}, t^{*}\right)$, if for every $\hat{t} \succeq t^{*}$ and for every $i, f^{i}$ satisfies MCS at $\left(x^{*}, t^{*}, \hat{t}\right)$. The following theorem is now easy to prove.

Theorem 3. Let $\Gamma$ be a parameterized game with strategic substitutes, $t^{*} \in T, x^{*} \in \mathcal{E}\left(t^{*}\right)$. If $\Gamma$ satisfies MCS at $\left(x^{*}, t^{*}\right)$, then there is $\hat{t} \succ t^{*}$ and there is $\hat{x}^{*} \in \mathcal{E}(\hat{t})$ such that $x^{*} \preceq \hat{x}^{*}$. If $\Gamma$ satisfies all parameter MCS at $\left(x^{*}, t^{*}\right)$, then for every $\hat{t} \succeq t^{*}$, there is $\hat{x}^{*} \in \mathcal{E}(\hat{t})$ such that $x^{*} \preceq \hat{x}^{*}$.

Proof. Suppose $\Gamma$ satisfies MCS at $\left(x^{*}, t^{*}\right)$. Then there is $\hat{t} \succ t^{*}$ such that for all $i, f^{i}$ satisfies MCS at $\left(x^{*}, t^{*}, \hat{t}\right)$. By the lemma above, for each $i, x_{i}^{*} \preceq g^{i}\left(\hat{y}_{-i}, \hat{t}\right)$, and therefore, $x^{*} \preceq g(\hat{y}, \hat{t})$, as required in Theorem 1 . The proof of the second statement is similar.

Theorem 3 provides for "local" monotone comparative statics 24 Conditions that guarantee "global" MCS can be posited as follows. A parameterized GSS $\Gamma$ satisfies $\boldsymbol{M C S}$, if for every $t^{*}$, there is $x^{*} \in \mathcal{E}\left(t^{*}\right)$ such that $\Gamma$ satisfies MCS at $\left(x^{*}, t^{*}\right)$. $\Gamma$ satisfies all parameter $\boldsymbol{M C S}$, if for every $t^{*}$, there is $x^{*} \in \mathcal{E}\left(t^{*}\right)$ such that $\Gamma$ satisfies all parameter MCS at $\left(x^{*}, t^{*}\right)$. The first two statements in the corollary below follow immediately. Still stronger results can be derived with stronger conditions, as follows. A parameterized game with strategic substitutes $\Gamma$ satisfies strong $\boldsymbol{M C S}$, if for every $t^{*}$ and for every $x^{*} \in \mathcal{E}\left(t^{*}\right), \Gamma$ satisfies MCS at $\left(x^{*}, t^{*}\right)$. $\Gamma$ satisfies all parameter strong $\boldsymbol{M C S}$, if for every $t^{*}$, and for every $x^{*} \in \mathcal{E}\left(t^{*}\right)$, $\Gamma$ satisfies all parameter MCS at $\left(x^{*}, t^{*}\right)$. The last two statements in the corollary below follow immediately.

Corollary 4. Let $\Gamma$ be a parameterized game with strategic substitutes.

(1) If $\Gamma$ satisfies MCS, then for every $t^{*} \in T$, there is $x^{*} \in \mathcal{E}\left(t^{*}\right)$, there is $\hat{t} \succ t^{*}$ and there is $\hat{x}^{*} \in \mathcal{E}(\hat{t})$ such that $x^{*} \preceq \hat{x}^{*}$.

(2) If $\Gamma$ satisfies all parameter $M C S$, then for every $t^{*} \in T$, there is $x^{*} \in \mathcal{E}\left(t^{*}\right)$, such that for every $\hat{t} \succeq t^{*}$, there is $\hat{x}^{*} \in \mathcal{E}(\hat{t})$ such that $x^{*} \preceq \hat{x}^{*}$.

(3) If $\Gamma$ satisfies strong $M C S$, then for every $t^{*} \in T$, and for every $x^{*} \in \mathcal{E}\left(t^{*}\right)$, there is $\hat{t} \succ t^{*}$ and there is $\hat{x}^{*} \in \mathcal{E}(\hat{t})$ such that $x^{*} \preceq \hat{x}^{*}$.

(4) If $\Gamma$ satisfies all parameter strong $M C S$, then for every $t^{*} \in T$, for every $x^{*} \in \mathcal{E}\left(t^{*}\right)$, for every $\hat{t} \succeq t^{*}$, there is $\hat{x}^{*} \in \mathcal{E}(\hat{t})$ such that $x^{*} \preceq \hat{x}^{*}$.

Notice that if for every $t$, the equilibrium is unique, then the strong MCS property is equivalent to MCS, and the all parameter strong MCS property is equivalent to all parameter MCS. An example in which all parameter strong MCS property holds is presented below.

\footnotetext{
${ }^{24}$ Notice that for twice continuously differentiable functions, the condition presented in section 2 can be translated to the MCS condition above.
} 


\section{Examples}

This section presents several examples. The Cournot duopoly game presented in section 2 is analyzed using the monotone comparative statics property mentioned above. The other two examples show applications of differentiability conditions mentioned in section 2 .

Example 1 (continued). Consider the Cournot duopoly example presented earlier. As shown below, for $\xi \in\left[\frac{1}{3}, \frac{2}{3}\right]$, this game satisfies all parameter MCS. Moreover, as equilibrium is unique, it satisfies all parameter strong MCS. Notice that the profit of each firm is

$$
\begin{aligned}
& f^{1}\left(x_{1}, x_{2}, t\right)=\left(a-b\left(x_{1}+x_{2}\right)\right) x_{1}-(c-\xi t) x_{1}, \text { and } \\
& f^{2}\left(x_{1}, x_{2}, t\right)=\left(a-b\left(x_{1}+x_{2}\right)\right) x_{2}-(c-(1-\xi) t) x_{2} .
\end{aligned}
$$

Fix $t^{*}$ and $\hat{t} \geq t^{*}$. Recall that $\left(x_{1}^{*}, x_{2}^{*}\right)=\left(\frac{a-c+(3 \xi-1) t^{*}}{3 b}, \frac{a-c+(2-3 \xi) t^{*}}{3 b}\right)$. It follows that for every $x_{1}, f^{1}\left(x_{1}, x_{2}^{*}, t^{*}\right)$ may be written as $f^{1}\left(x_{1}, x_{2}^{*}, t^{*}\right)=\left[\frac{2(a-c)}{3}-b x_{1}+\frac{2(3 \xi-1) t^{*}}{3}\right] x_{1}$. Moreover, recall that $\hat{y}_{2}=g^{2}\left(x_{1}^{*}, \hat{t}\right)=\frac{a-c+(1-\xi) \hat{t}-b x_{1}^{*}}{2 b}$, and this simplifies to $\hat{y}_{2}=\frac{a-c}{3 b}+\frac{(1-\xi) \hat{t}}{2 b}-\frac{(3 \xi-1) t^{*}}{6 b}$. Using this $\hat{y}_{2}$, notice that for every $x_{1}, f^{1}\left(x_{1}, \hat{y}_{2}, \hat{t}\right)$ may be written as $f^{1}\left(x_{1}, \hat{y}_{2}, \hat{t}\right)=f^{1}\left(x_{1}, y_{2}^{*}, t^{*}\right)+$ $\frac{(3 \xi-1)\left(\hat{t}-t^{*}\right)}{2} x_{1}$.

Now suppose $x_{1} \geq x_{1}^{\prime}$, and $f^{1}\left(x_{1}, x_{2}^{*}, t^{*}\right) \geq f^{1}\left(x_{1}^{\prime}, x_{2}^{*}, t^{*}\right)$. Then it can be calculated that $f^{1}\left(x_{1}, \hat{y}_{2}, \hat{t}\right) \geq f^{1}\left(x_{1}^{\prime}, \hat{y}_{2}, \hat{t}\right)$, if, and only if $\frac{(3 \xi-1)\left(\hat{t}-t^{*}\right)}{2} x_{1} \geq \frac{(3 \xi-1)\left(\hat{t}-t^{*}\right)}{2} x_{1}^{\prime}$, and this holds, if $\xi \geq \frac{1}{3}$. Similarly, it can be shown that the condition for player 2 holds, if $\xi \leq \frac{2}{3}$.

Example 2. Consider a common-pool resource game25 Consider two players, indexed $i=1,2$, each with an endowment $e_{i}>0$. There are two investment options: (1) a common resource (such as a fishery) that exhibits diminishing marginal return, and (2) a (potentially asymmetric) outside option with constant marginal return, $r_{i}>0$. If player $i$ invests an amount $x_{i} \leq e_{i}$ of his endowment into the common resource, he receives a proportional share of total output $\left(a\left(x_{1}+x_{2}\right)-b\left(x_{1}+x_{2}\right)^{2}\right)$. Thus, payoff to player $i$ is given by

$$
f^{i}\left(x_{1}, x_{2}\right)=r_{i}\left(e_{i}-x_{i}\right)+\frac{x_{i}}{x_{1}+x_{2}}\left(a\left(x_{1}+x_{2}\right)-b\left(x_{1}+x_{2}\right)^{2}\right) .
$$

It is well-known that in a Nash equilibrium, the common-pool resource is over-appropriated, and this has a long-run implication for resource conservation. A question of interest is whether a regulator can provide decentralized tax incentives to induce both players to reduce their individual investment in the common pool resource, and thereby reduce overappropriation. Equivalently, under what conditions will a subsidy increase individual investment in the common-pool resource? Consider the following subsidy-parameterized payoff for player $i$.

$$
f^{i}\left(x_{1}, x_{2}, t\right)=(1-t) r_{i}\left(e_{i}-x_{i}\right)+\frac{x_{i}}{x_{1}+x_{2}}\left(a\left(x_{1}+x_{2}\right)-b\left(x_{1}+x_{2}\right)^{2}\right) .
$$

It is easy to calculate that $f_{1, t}^{1}=r_{1}, f_{1,2}^{1}=-b, f_{2, t}^{2}=r_{2}$, and $f_{2,2}^{2}=-2 b$. Consequently, $f_{1, t}^{1}+\left.f_{1,2}^{1}\left(-\frac{f_{2, t}^{2}}{f_{2,2}^{2}}\right)\right|_{\left(x^{*}, t^{*}\right)} \geq 0 \Leftrightarrow 2 r_{1} \geq r_{2}$. Similarly, $f_{2, t}^{2}+\left.f_{1,2}^{2}\left(-\frac{f_{1, t}^{1}}{f_{1,1}^{1}}\right)\right|_{\left(x^{*}, t^{*}\right)}>0 \Leftrightarrow$

\footnotetext{
${ }^{25}$ See, for example, Ostrom, Gardner, and Walker (1994).
} 
$r_{2} \geq \frac{r_{1}}{2}$. It follows that equilibria increase in the cone determined by $r_{2}=2 r_{1}$ and $r_{2}=\frac{1}{2} r_{1}$ in the $\left(r_{1}, r_{2}\right)$-space.

Example 3. Consider a game of tournaments 26 Suppose a tournament has 3 players, where a parameterized reward $r(t)$ (with $0 \leq t \leq T$, and $r^{\prime}(t)>0$ ) is shared by the players who succeed in the tournament. If one player succeeds, he gets $r(t)$ for sure, if two players succeed, each gets $r(t)$ with probability one-half, and if all players succeed, each gets $r(t)$ with probability one-third. Each player chooses effort $x_{i} \in[0,1]$ with probability of success $x_{i}$. Expected reward per unit for player $i$ is

$$
\pi^{i}\left(x_{i}, x_{j}, x_{k}\right)=x_{i}\left(1-x_{j}\right)\left(1-x_{k}\right)+\frac{1}{2} x_{i} x_{j}\left(1-x_{k}\right)+\frac{1}{2} x_{i} x_{k}\left(1-x_{j}\right)+\frac{1}{3} x_{i} x_{j} x_{k} .
$$

The quadratic cost of effort $x_{i}$ is $\frac{c_{i}}{2} x_{i}^{2}$, and is allowed to be asymmetric across players. The payoff to player $i$ is expected reward minus cost of effort. That is,

$$
f^{i}\left(x_{i}, x_{j}, x_{k}, t\right)=r(t) \pi^{i}\left(x_{i}, x_{j}, x_{k}\right)-\frac{c_{i}}{2} x_{i}^{2},
$$

Here's a question of interest: if tournament organizers increase reward, when will all players compete more strongly?27 Notice that $f_{i, j}^{i}=r(t) \pi_{i, j}^{i}\left(x_{k}\right), f_{i, k}^{i}=r(t) \pi_{i, k}^{i}\left(x_{j}\right), f_{i, t}^{i}=$ $r^{\prime}(t) \pi_{i}^{i}\left(x_{j}, x_{k}\right) ;-\frac{f_{j, t}^{j}}{f_{j, j}^{j}}=\frac{r^{\prime}(t) \pi_{j}^{j}\left(x_{i}, x_{k}\right)}{c_{j}},-\frac{f_{k, t}^{k}}{f_{k, k}^{k}}=\frac{r^{\prime}(t) \pi_{k}^{k}\left(x_{i}, x_{j}\right)}{c_{k}} ;$ and $x_{i}^{*}=\frac{r\left(t^{*}\right) \pi_{i}^{i}\left(x_{j}^{*}, x_{k}^{*}\right)}{c_{i}}, x_{j}^{*}=\frac{r\left(t^{*}\right) \pi_{j}^{j}\left(x_{i}^{*}, x_{k}^{*}\right)}{c_{j}}$, $x_{k}^{*}=\frac{r\left(t^{*}\right) \pi_{k}^{k}\left(x_{i}^{*}, x_{j}^{*}\right)}{c_{k}}$. Therefore,

$$
\begin{aligned}
& f_{i, t}^{i}+f_{i, j}^{i}\left(-\frac{f_{j, t}^{j}}{f_{j, j}^{j}}\right)+\left.f_{i, k}^{i}\left(-\frac{f_{k, t}^{k}}{f_{k, k}^{k}}\right)\right|_{\left(x^{*}, t^{*}\right)}>0 \\
\Leftrightarrow & r^{\prime}(t) \pi_{i}^{i}\left(x_{j}, x_{k}\right)+r^{\prime}(t) \pi_{i, j}^{i}\left(x_{k}\right) x_{j}+\left.r^{\prime}(t) \pi_{i, k}^{i}\left(x_{j}\right) x_{k}\right|_{\left(x^{*}, t^{*}\right)}>0 \\
\Leftrightarrow & \pi_{i}^{i}\left(x_{j}^{*}, x_{k}^{*}\right)+\pi_{i, j}^{i}\left(x_{k}^{*}\right) x_{j}^{*}+\pi_{i, k}^{i}\left(x_{j}^{*}\right) x_{k}^{*}>0 .
\end{aligned}
$$

Moreover,

$$
\begin{aligned}
& \pi_{i}^{i}\left(x_{j}^{*}, x_{k}^{*}\right)+\pi_{i, j}^{i}\left(x_{k}^{*}\right) x_{j}^{*}+\pi_{i, k}^{i}\left(x_{j}^{*}\right) x_{k}^{*} \\
= & \left(1-x_{j}^{*}\right)\left(1-x_{k}^{*}\right)+\frac{1}{2}\left(x_{j}^{*}\left(1-x_{k}^{*}\right)+x_{k}^{*}\left(1-x_{j}^{*}\right)\right)+\frac{1}{3} x_{j}^{*} x_{k}^{*} \\
& \quad+\left[\frac{1}{3} x_{k}^{*}-\frac{1}{2}\left(\left(1-x_{k}^{*}\right)+x_{k}^{*}\right)\right] x_{j}^{*}+\left[\frac{1}{3} x_{j}^{*}-\frac{1}{2}\left(\left(1-x_{j}^{*}\right)+x_{j}^{*}\right)\right] x_{k}^{*} \\
= & \left(1-x_{j}^{*}\right)\left(1-x_{k}^{*}\right) .
\end{aligned}
$$

Therefore, the condition on payoff functions is satisfied, if each of $x_{i}^{*}, x_{j}^{*}, x_{k}^{*}$ is less than 1 . In other words, if the equilibrium is not degenerate, that is, no player wins the tournament for sure, then the equilibrium increases with the parameter. The result extends to $N$ player tournaments, as shown in the appendix.

Similar applications can be made to other games as well. In particular, an application of the differentiability results does not necessarily require knowledge of best-response functions, or closed form solutions for an equilibrium. Therefore, from a practical point of view, the results here can have broad applications.

\footnotetext{
${ }^{26}$ This version is based on Dubey, Haimanko, and Zapechelnyuk (2006).

${ }^{27}$ Tournament organizers might have an incentive to have players compete more strongly, perhaps because it increases audience size, and therefore, ticket sales.
} 


\section{$5 \quad$ Extensions to Correspondences}

The next subsection extends theorem 1 to the case of correspondences, and the subsection after that extends theorem 3 to the case of correspondences.

\subsection{Conditions on Correspondences}

Consider a triple $(X, T, g)$, where $(X, \preceq)$ is a nonempty, subcomplete, sublattice of a Banach lattice, $T$ is a nonempty partially ordered set, and $g: X \times T \rightarrow X$ is a correspondence. For a triple $(X, T, g)$, consider the following sets of assumptions.

Assumption II.A: For every $x \in X, g(x, \cdot)$ is nondecreasing in $t 28$ and for every $t \in T$, $g(\cdot, t)$ is nonincreasing in $x$, and nonempty-sublattice-valued 29

Assumpton II.B: $(X, \preceq)$ is a nonempty, compact, convex, sublattice of a Banach lattice; order intervals in $X$ are closed and convex; and for every $t, g(\cdot, t)$ is upper hemi-continuous, and nonempty-compact-convex valued.

Assumption II.C: $(X, \preceq)$ is a nonempty, closed, convex sublattice of a Banach lattice; order intervals in $X$ are closed and convex; for every $t, g(X, t)$ is relatively compact; and for every $t, g(\cdot, t)$ is upper hemi-continuous, and nonempty-closed-convex-valued.

As earlier, a triple $(X, T, g)$ is admissible-II if it satisfies either II.A and II.B, or II.A and II.C. For each $t$, let $\mathcal{E}(t)=\{x \in X \mid x \in g(x, t)\}$ be the equilibrium (or fixed) points of $g$ at $t$. Theorems of Kakutani-Glicksberg-Ky Fan, or Bohnenlust-Karlin imply that for every $t, \mathcal{E}(t)$ is non-empty. The following extends theorem 1 to correspondences 30

Theorem 4. Let $(X, T, g)$ be an admissible-II triple. Fix $t^{*} \in T$, and let $x^{*} \in \mathcal{E}\left(t^{*}\right)$. Consider $\hat{t} \in T$ such that $t^{*} \preceq \hat{t}$, and let $\hat{y}=\sup _{X} g\left(x^{*}, \hat{t}\right)$.

If $x^{*} \preceq i n f_{X} g(\hat{y}, \hat{t})$, then there is $\hat{x}^{*} \in \mathcal{E}(\hat{t})$ such that $x^{*} \preceq \hat{x}^{*}$.

Proof. Notice that $x^{*} \preceq \hat{y}$, because $g$ is nondecreasing in $t$, (hence $g\left(x^{*}, t^{*}\right) \sqsubseteq g\left(x^{*}, \hat{t}\right)$, ) $x^{*} \in g\left(x^{*}, t^{*}\right)$, and $\sup g\left(x^{*}, t^{*}\right) \preceq \hat{y}$. Moreover, for every $x$ in $\left[x^{*}, \hat{y}\right], g(x, \hat{t}) \subset\left[x^{*}, \hat{y}\right]$, and this can be seen as follows. Suppose $x^{*} \preceq x \preceq \hat{y}$. Then $x \preceq \hat{y}$ implies that inf $g(x, \hat{t}) \succeq$ $\inf g(\hat{y}, \hat{t}) \succeq x^{*}$, where the first inequality follows from the fact that $g(\cdot, \hat{t})$ is weakly decreasing with respect to $\sqsubseteq$, and the second follows from the condition in the proposition. Moreover, $x^{*} \preceq x$ implies that $\sup g(x, \hat{t}) \preceq \sup g\left(x^{*}, \hat{t}\right)=\hat{y}$, where the inequality follows from weakly decreasing $g(\cdot, \hat{t})$, and the equality follows from definition of $\hat{y}$. Therefore, the restriction of $g(\cdot, \hat{t})$ to $\left[x^{*}, \hat{y}\right]$ is a correspondence from $\left[x^{*}, \hat{y}\right]$ to $\left[x^{*}, \hat{y}\right]$. By Kakutani-Glicksberg-Ky Fan or by Bohnenlust-Karlin, there is $\hat{x}^{*} \in\left[x^{*}, \hat{y}\right]$ such that $\hat{x}^{*} \in g\left(\hat{x}^{*}, \hat{t}\right)$. Consequently, there is $\hat{x}^{*} \in \mathcal{E}(\hat{t})$ such that $x^{*} \preceq \hat{x}^{*}$.

\footnotetext{
${ }^{28}$ For every $t, \hat{t} \in T, t \preceq \hat{t} \Rightarrow$ for every $x, g(x, t) \sqsubseteq g(x, \hat{t})$.

${ }^{29}$ For every $x, y \in X, x \preceq y \Rightarrow$ for every $t, g(y, t) \sqsubseteq g(x, t)$.

${ }^{30} \mathrm{We}$ are grateful to an anonymous referee for pointing out an earlier version of this extension.
} 


\subsection{Conditions on General Payoff Functions}

Consider the following definitions. For non-empty subsets $A, B$ of $X, A$ is completely lower than $B$, denoted $A \sqsubseteq_{c} B$ if for every $a \in A$, and for every $b \in B, a \preceq b$. Let $\Gamma=\left(I, T,\left(X^{i}, \preceq^{i}\right.\right.$ ,$\left.f^{i}\right)_{i \in I}$ ) be a parameterized game with strategic substitutes. Suppose for each $i, f^{i}$ is strictly quasi-supermodular 31 and for each $\left(x_{-i}, t\right)$, $\arg \max _{x_{i}} f^{i}\left(x_{i}, x_{-i}, t\right)$ is convex-valued. Let $g^{i}\left(x_{-i}, t\right)=\arg \max _{x_{i}} f^{i}\left(x_{i}, x_{-i}, t\right)$, and $g=\left(g^{i}\right)_{i \in I}$. In this case, let $t^{*} \in T, x^{*} \in \mathcal{E}\left(t^{*}\right)$, and let $\hat{t} \succ t^{*}$. For player $i, f^{i}$ satisfies monotone comparative statics (MCS)-II at $\left(x^{*}, t^{*}, \hat{t}\right)$, if for every $x_{i} \succeq x_{i}^{\prime}, f^{i}\left(x_{i}, x_{-i}^{*}, t^{*}\right) \geq f^{i}\left(x_{i}^{\prime}, x_{-i}^{*}, t^{*}\right) \Rightarrow f^{i}\left(x_{i}, \hat{y}_{-i}, \hat{t}\right)>f^{i}\left(x_{i}^{\prime}, \hat{y}_{-i}, \hat{t}\right)$, where $\hat{y}_{-i}=\sup g_{-i}\left(x^{*}, \hat{t}\right)$ 32 Here is a corresponding lemma.

Lemma 2. If $f^{i}$ satisfies MCS-II at $\left(x^{*}, t^{*}, \hat{t}\right)$, then $x_{i}^{*} \preceq \inf g^{i}\left(\hat{y}_{-i}, \hat{t}\right)$.

Proof. Fix $t^{*} \in T, x^{*} \in \mathcal{E}\left(t^{*}\right), \hat{t} \succ t^{*}$, and let $\hat{y}_{-i}=\sup g_{-i}\left(x^{*}, \hat{t}\right)$. Notice that $g^{i}\left(x_{-i}^{*}, t^{*}\right) \sqsubseteq_{c}$ $g^{i}\left(\hat{y}_{-i}, \hat{t}\right)$, as follows. Fix $x_{i} \in g^{i}\left(x_{-i}^{*}, t^{*}\right)$ and $\hat{x}_{i} \in g^{i}\left(\hat{y}_{-i}, \hat{t}\right)$ arbitrarily. Suppose $x_{i}$ and $\hat{x}_{i}$ are unordered. Then $f^{i}\left(x_{i}, x_{-i}^{*}, t^{*}\right) \geq f^{i}\left(x_{i} \wedge \hat{x}_{i}, x_{-i}^{*}, t^{*}\right)$, and therefore, using strict quasisupermodularity, $f^{i}\left(x_{i} \vee \hat{x}_{i}, x_{-i}^{*}, t^{*}\right)>f^{i}\left(\hat{x}_{i}, x_{-i}^{*}, t^{*}\right)$, and therefore, using MCS-II at $\left(x^{*}, t^{*}, \hat{t}\right)$, $f^{i}\left(x_{i} \vee \hat{x}_{i}, \hat{y}_{-i}, \hat{t}\right)>f^{i}\left(\hat{x}_{i}, \hat{y}_{-i}, \hat{t}\right)$, a contradiction. Thus, $x_{i}$ and $\hat{x}_{i}$ are ordered. Suppose $x_{i} \npreceq \hat{x}_{i}$. Then $\hat{x}_{i} \prec x_{i}$. Moreover, $f^{i}\left(x_{i}, x_{-i}^{*}, t^{*}\right) \geq f^{i}\left(\hat{x}_{i}, x_{-i}^{*}, t^{*}\right)$, and therefore, using MCSII at $\left(x^{*}, t^{*}, \hat{t}\right), f^{i}\left(x_{i}, \hat{y}_{-i}, \hat{t}\right)>f^{i}\left(\hat{x}_{i}, \hat{y}_{-i}, \hat{t}\right)$, a contradiction. Thus, $x_{i} \preceq \hat{x}_{i}$, as desired. Now, $g^{i}\left(x_{-i}^{*}, t^{*}\right) \bigsqcup_{c} g^{i}\left(\hat{y}_{-i}, \hat{t}\right)$ implies that $x_{i}^{*} \preceq \sup g^{i}\left(x_{-i}^{*}, t^{*}\right) \preceq \inf g^{i}\left(\hat{y}_{-i}, \hat{t}\right)$, as desired.

Let $\Gamma$ be a parameterized game with strategic substitutes, $t^{*} \in T$, and $x^{*} \in \mathcal{E}\left(t^{*}\right)$. $\Gamma$ satisfies $\boldsymbol{M C S}-\boldsymbol{I I}$ at $\left(x^{*}, t^{*}\right)$, if there is $\hat{t} \succ t^{*}$ such that for all $i, f^{i}$ satisfies MCS-II at $\left(x^{*}, t^{*}, \hat{t}\right)$. $\Gamma$ satisfies all parameter MCS-II at $\left(x^{*}, t^{*}\right)$, if for every $\hat{t} \succ t^{*}$ and for every $i, f^{i}$ satisfies MCS-II at $\left(x^{*}, t^{*}, \hat{t}\right)$. Lemma 2 and theorem 4 yield the following theorem.

Theorem 5. Let $\Gamma$ be a parameterized game with strategic substitutes, $t^{*} \in T, x^{*} \in \mathcal{E}\left(t^{*}\right)$. If $\Gamma$ satisfies MCS-II at $\left(x^{*}, t^{*}\right)$, then there is $\hat{t} \succ t^{*}$ and there is $\hat{x}^{*} \in \mathcal{E}(\hat{t})$ such that $x^{*} \preceq \hat{x}^{*}$. If $\Gamma$ satisfies all parameter MCS-II at $\left(x^{*}, t^{*}\right)$, then for every $\hat{t} \succeq t^{*}$, there is $\hat{x}^{*} \in \mathcal{E}(\hat{t})$ such that $x^{*} \preceq \hat{x}^{*}$.

As earlier, this can be viewed as a "local" monotone comparative statics. Conditions that guarantee "global" MCS can be posited, too. A parameterized game with strategic substitutes $\Gamma$ satisfies $\boldsymbol{M C S}-\boldsymbol{I I}$, if for every $t^{*}$, there is $x^{*} \in \mathcal{E}\left(t^{*}\right)$ such that $\Gamma$ satisfies MCS-II at $\left(x^{*}, t^{*}\right)$. $\Gamma$ satisfies all parameter $\boldsymbol{M C S}$-II , if for every $t^{*}$, there is $x^{*} \in \mathcal{E}\left(t^{*}\right)$ such that $\Gamma$ satisfies all parameter MCS-II at $\left(x^{*}, t^{*}\right)$. Similarly, a strong MCS-II and an all parameter strong MCS-II property can be posited, and a corollary similar to corollary 4 holds.

\footnotetext{
${ }^{31}$ As usual, a function $f: X \rightarrow \mathbb{R}$ is strictly quasisupermodular, if for all unordered $x, y \in X, f(x) \geq$ $f(x \wedge y) \Longrightarrow f(x \vee y)>f(y)$.

${ }^{32}$ As earlier, this can be viewed as a restricted version of a strict single-crossing property, as defined in Shannon (1995). Moreover, as earlier, an alternative condition can be postulated requiring only an upper bound for the best-response set.
} 


\section{References}

Aliprantis, C. D., And K. C. Border (1994): Infinite Dimensional Analysis: A Hitchhiker's Guide. Springer-Verlag.

AmiR, R. (1996): "Cournot Oligopoly and the Theory of Supermodular Games," Games and Economic Behavior, 15, 132-148.

Amir, R., And V. E. Lambson (2000): "On the Effects of Entry in Cournot Markets," The Review of Economic Studies, 67(2), 235-254.

Bulow, J. I., J. D. Geanakoplos, and P. D. Klemperer (1985): "Multimarket Oligopoly: Strategic Substitutes and Complements," Journal of Political Economy, 93(3), $488-511$.

DAcic, R. M. (1981): "Properties of Monotone Mappings in Partially Ordered Sets," Publications De L'Institut Mathematique, 30, 33-39.

Dubey, P., O. Haimanko, and A. Zapechelnyuk (2006): "Strategic complements and substitutes, and potential games," Games and Economic Behavior, 54, 77-94.

ECHENIqUe, F. (2002): "Comparative statics by adaptive dynamics and the correspondence principle," Econometrica, 70(2), 257-289.

Echenique, F., And T. Sabarwal (2003): "Strong Comparative Statics of Equilibria," Games and Economic Behavior, 42(2), 307-314.

Edlin, A., And C. Shannon (1998): "Strict Monotonicity in Comparative Statics," Journal of Economic Theory, 81(1), 201-219.

Jensen, M. K. (2010): "Aggregative games and best-reply potentials," Economic Theory, p. forthcoming.

Lippman, S. A., J. W. Mamer, and K. F. MCCArdle (1987): "Comparative Statics in non-cooperative games via transfinitely iterated play," Journal of Economic Theory, 41(2), 288-303.

Milgrom, P., And J. Roberts (1990): "Rationalizability, learning, and equilibrium in games with strategic complementarities," Econometrica, 58(6), 1255-1277.

(1994): “Comparing Equilibria," American Economic Review, 84(3), 441-459.

Milgrom, P., and C. Shannon (1994): "Monotone Comparative Statics," Econometrica, $62(1), 157-180$.

Ostrom, E., R. Gardner, And J. Walker (1994): Rules, Games, and Common-Pool Resources. The University of Michigan Press, Ann Arbor.

Quah, J. K.-H. (2007): "The Comparative Statics of Constrained Optimization Problems," Econometrica, 75(2), 401-431. 
Roy, S. (2002): "A Note on Comparative Statics of Fixed Points of Non-monotone Mappings," in Labor Contracts under General Equilibrium: Three Essays on the Comparative Statics of Employment, Ph.D. Dissertation, University of Southern California.

Roy, S., And T. SABARWAL (2008): "On the (Non-)Lattice Structure of the Equilibrium Set in Games With Strategic Substitutes," Economic Theory, 37(1), 161-169.

Shannon, C. (1995): "Weak and Strong Monotone Comparative Statics," Economic Theory, 5(2), 209-227.

Sobel, J. (1988): "Isotone comparative statics in supermodular games," Mimeo. SUNY at Stony Brook.

TARski, A. (1955): "A Lattice-theoretical Fixpoint Theorem and its Application," Pacific Journal of Mathematics, 5(2), 285-309.

Topkis, D. (1978): "Minimizing a submodular function on a lattice," Operations Research, $26,305-321$.

(1979): "Equilibrium points in nonzero-sum $n$-person submodular games," SIAM Journal on Control and Optimization, 17(6), 773-787.

_ (1998): Supermodularity and Complementarity. Princeton University Press.

Villas-Boas, J. M. (1997): "Comparative Statics of Fixed Points," Journal of Economic Theory, 73(1), 183-198.

Vives, X. (1990): "Nash Equilibrium with Strategic Complementarities," Journal of Mathematical Economics, 19(3), 305-321.

— (1999): Oligopoly Pricing. MIT Press.

— (2005): "Complementarities and Games: New Developments," Journal of Economic Literature, 43(2), 437-479.

Zhou, L. (1994): "The Set of Nash Equilibria of a Supermodular Game is a Complete Lattice," Games and Economic Behavior, 7(2), 295-300. 


\section{Appendix}

Example 3 (continued). Suppose a tournament has $N \geq 2$ players, where a parameterized reward $r(t)$ (with $0 \leq t \leq T$, and $r^{\prime}(t)>0$ ) is shared by the players who succeed in the tournament. If one player succeeds, she gets $r(t)$ for sure, if two players succeed, each gets $r(t)$ with probability one-half, and if all players succeed, each gets $r(t)$ with probability one-third. For player $i=1$, the expected reward per unit is

$$
\begin{aligned}
\pi^{1}\left(x_{1}, \ldots, x_{N}\right)= & x_{1} \prod_{i_{1} \in\{2, \ldots, N\}}\left(1-x_{i_{1}}\right) \\
& +\frac{1}{2} \sum_{i_{1}=2}^{N} x_{1} x_{i_{1}} \prod_{i_{2} \in\{2, \ldots, N\} \backslash\left\{i_{1}\right\}}\left(1-x_{i_{2}}\right) \\
& +\frac{1}{3} \sum_{i_{1}=2}^{N} \sum_{i_{2}=i_{1}+1}^{N} x_{1} x_{i_{1}} x_{i_{2}} \prod_{i_{3} \in\{2, \ldots, N\} \backslash\left\{i_{1}, i_{2}\right\}}\left(1-x_{i_{3}}\right) \\
& +\frac{1}{4} \sum_{i_{1}=2}^{N} \sum_{i_{2}=i_{1}+1}^{N} \sum_{i_{3}=i_{2}+1}^{N} x_{1} x_{i_{1}} x_{i_{2}} x_{i_{3}} \prod_{i_{4} \in\{2, \ldots, N\} \backslash\left\{i_{1}, i_{2}, i_{3}\right\}}\left(1-x_{i_{4}}\right) \\
& +\ldots \\
& +\frac{1}{N} x_{1} x_{2} \cdots x_{N},
\end{aligned}
$$

and the expected reward is $r(t) \pi^{i}\left(x_{1}, \ldots, x_{N}\right)$. The quadratic cost of effort $x_{1}$ is $\frac{c_{1}}{2} x_{1}^{2}$, and it is allowed to be asymmetric across players. The payoff to player 1 is expected reward minus cost of effort. That is,

$$
f^{1}\left(x_{1}, \ldots, x_{N}\right)=r(t) \pi^{1}\left(x_{1}, \ldots, x_{N}\right)-\frac{c_{1}}{2} x_{1}^{2} .
$$

Following the same argument as in the text, it follows that

$$
f_{1, t}^{1}+\left.\sum_{n=2}^{N} f_{1, n}^{1}\left(-\frac{f_{n, t}^{n}}{f_{n, n}^{n}}\right)\right|_{\left(x^{*}, t^{*}\right)}>0 \Leftrightarrow \pi_{1}^{1}\left(x_{-1}^{*}\right)+\sum_{n=2}^{N} x_{n}^{*} \pi_{1, n}^{1}\left(x_{-(1, n)}^{*}\right)>0 .
$$

Here, as usual, $x_{-(1, n)}^{*}$ is the vector $x^{*}$ without components 1 and $n$. The details below show that

$$
\pi_{1}^{1}\left(x_{-1}^{*}\right)+\sum_{n=2}^{N} x_{n}^{*} \pi_{1, n}^{1}\left(x_{-(1, n)}^{*}\right)=\prod_{n=2}^{N}\left(1-x_{n}^{*}\right)
$$

A similar result holds for each player $i$, and therefore, it follows that if the equilibrium is not degenerate, then equilibrium increases with the parameter.

Details. Notice that

$$
\begin{aligned}
\pi_{1}^{1}\left(x_{-1}^{*}\right)= & \prod_{i_{1} \in\{2, \ldots, N\}}\left(1-x_{i_{1}}^{*}\right) \\
& +\frac{1}{2} \sum_{i_{1}=2}^{N} x_{i_{1}}^{*} \prod_{i_{2} \in\{2, \ldots, N\} \backslash\left\{i_{1}\right\}}\left(1-x_{i_{2}}^{*}\right) \\
& +\frac{1}{3} \sum_{i_{1}=2}^{N} \sum_{i_{2}=i_{1}+1}^{N} x_{i_{1}}^{*} x_{i_{2}}^{*} \prod_{i_{3} \in\{2, \ldots, N\} \backslash\left\{i_{1}, i_{2}\right\}}\left(1-x_{i_{3}}^{*}\right) \\
& +\frac{1}{4} \sum_{i_{1}=2}^{N} \sum_{i_{2}=i_{1}+1}^{N} \sum_{i_{3}=i_{2}+1}^{N} x_{i_{1}}^{*} x_{i_{2}}^{*} x_{i_{3}}^{*} \prod_{i_{4} \in\{2, \ldots, N\} \backslash\left\{i_{1}, i_{2}, i_{3}\right\}}\left(1-x_{i_{4}}^{*}\right) \\
& +\ldots \\
& +\frac{1}{N} x_{2}^{*} \cdots x_{N} .
\end{aligned}
$$


Moreover, for each $n=2, \ldots, N$,

$$
\begin{aligned}
\pi_{1, n}^{1}\left(x_{-(1, n)}^{*}\right)= & -\prod_{i_{1} \in\{2, \ldots, N\} \backslash\{n\}}\left(1-x_{i_{1}}^{*}\right) \\
& +\frac{1}{2} \prod_{i_{2} \in\{2, \ldots, N\} \backslash\{n\}}\left(1-x_{i_{2}}^{*}\right) \\
& -\frac{1}{2} \sum_{i_{1} \in\{2, \ldots, N\} \backslash\{n\}} x_{i_{1}}^{*} \prod_{i_{2} \in\{2, \ldots, N\} \backslash\left\{i_{1}, n\right\}}\left(1-x_{i_{2}}^{*}\right) \\
& +\frac{1}{3} \sum_{i_{2} \in\{2, \ldots, N\} \backslash\{n\}} x_{i_{2}}^{*} \prod_{i_{3} \in\{2, \ldots, N\} \backslash\left\{i_{2}, n\right\}}\left(1-x_{i_{3}}^{*}\right) \\
& -\frac{1}{3} \sum_{i_{1} \in\{2, \ldots, N\} \backslash\{n\}} x_{i_{2} \in\left\{i_{1}+1, \ldots, N\right\} \backslash\{n\}} x_{i_{1}}^{*} x_{i_{2}}^{*} \prod_{i_{3} \in\{2, \ldots, N\} \backslash\left\{i_{1}, i_{2}, n\right\}}\left(1-x_{i_{3}}^{*}\right) \\
& +\frac{1}{4} \sum_{i_{2} \in\{2, \ldots, N\} \backslash\{n\}} \sum_{i_{3} \in\left\{i_{2}+1, \ldots, N\right\} \backslash\{n\}} x_{i_{2}}^{*} x_{i_{3}}^{*} \prod_{i_{4} \in\{2, \ldots, N\} \backslash\left\{i_{2}, i_{3}, n\right\}}\left(1-x_{i_{4}}^{*}\right) \\
& -\frac{1}{4} \sum_{i_{1} \in\{2, \ldots, N\} \backslash\{n\}} \sum_{i_{2} \in\left\{i_{1}+1, \ldots, N\right\} \backslash\{n\}} \sum_{i_{3} \in\left\{i_{2}+1, \ldots, N\right\} \backslash\{n\}} \sum_{i_{1}}^{*} x_{i_{2}}^{*} x_{i_{3}}^{*} \prod_{i_{4} \in\{2, \ldots, N\} \backslash\left\{i_{1}, i_{2}, i_{3}, n\right\}}\left(1-x_{i_{4}}^{*}\right) \\
& + \\
& +\frac{1}{N} \prod_{i_{1} \in\{2, \ldots, N\} \backslash\{n\}} x_{i_{1}}^{*} .
\end{aligned}
$$

Using the above expression for $\pi_{1, n}^{1}\left(x_{-(1, n)}^{*}\right)$, notice that the first term in $x_{n}^{*} \pi_{1, n}^{1}\left(x_{-(1, n)}^{*}\right)$, the one that has a coefficient of -1 , is $-x_{n}^{*} \prod_{i_{1} \in\{2, \ldots, N\} \backslash\{n\}}\left(1-x_{i_{1}}^{*}\right)$, and therefore, the sum of such terms, as $n$ varies over $2, \ldots, N$, can be written as

$$
-\sum_{i_{1} \in\{2, \ldots, N\}} x_{i_{1}}^{*} \prod_{i_{2} \in\{2, \ldots, N\} \backslash\left\{i_{1}\right\}}\left(1-x_{i_{2}}^{*}\right) .
$$

Similarly, notice that the term in $x_{n}^{*} \pi_{1, n}^{1}\left(x_{-(1, n)}^{*}\right)$ that has a coefficient of $+\frac{1}{2}$ is $\frac{1}{2} x_{n}^{*} \prod_{i_{2} \in\{2, \ldots, N\} \backslash\{n\}}\left(1-x_{i_{2}}^{*}\right)$, and therefore, the sum of such terms, as $n$ varies over $2, \ldots, N$, can be written as

$$
\frac{1}{2} \sum_{i_{1} \in\{2, \ldots, N\}} x_{i_{1}}^{*} \prod_{i_{2} \in\{2, \ldots, N\} \backslash\left\{i_{1}\right\}}\left(1-x_{i_{2}}^{*}\right) .
$$

Now notice that the sum of the previous two sums cancels the second term in the expression for $\pi_{1}^{1}\left(x_{-1}^{*}\right)$, the one with coefficient $\frac{1}{2}$.

Similarly, it can be calculated that the term in $x_{n}^{*} \pi_{1, n}^{1}\left(x_{-(1, n)}^{*}\right)$ that has a coefficient of $-\frac{1}{2}$, when summed as $n$ varies over $2, \ldots, N$, can be written as

$$
\begin{aligned}
& -\frac{1}{2} \sum_{n=2}^{N} \sum_{i_{1} \in\{2, \ldots, N\} \backslash\{n\}} x_{n}^{*} x_{i_{1}}^{*} \prod_{i_{2} \in\{2, \ldots, N\} \backslash\left\{i_{1}, n\right\}}\left(1-x_{i_{2}}^{*}\right) \\
& \quad=-\sum_{i_{1}=2}^{N} \sum_{i_{2}=i_{1}+1}^{N} x_{i_{1}}^{*} x_{i_{2}}^{*} \prod_{i_{3} \in\{2, \ldots, N\} \backslash\left\{i_{1}, i_{2}\right\}}\left(1-x_{i_{3}}^{*}\right),
\end{aligned}
$$

where the equality follows from adding terms that appear exactly two times. In the same manner, the term in $x_{n}^{*} \pi_{1, n}^{1}\left(x_{-(1, n)}^{*}\right)$ that has a coefficient of $+\frac{1}{3}$, when summed as $n$ varies over $2, \ldots, N$, can be written, after adding terms that appear exactly two times, as

$$
\frac{2}{3} \sum_{i_{1}=2}^{N} \sum_{i_{2}=i_{1}+1}^{N} x_{i_{1}}^{*} x_{i_{2}}^{*} \prod_{i_{3} \in\{2, \ldots, N\} \backslash\left\{i_{1}, i_{2}\right\}}\left(1-x_{i_{3}}^{*}\right) .
$$

Notice again that the sum of the last two double sums cancels the third term in the expression for $\pi_{1}^{1}\left(x_{-1}^{*}\right)$, the one with coefficient $\frac{1}{3}$. Similarly, it can be concluded that

$$
\pi_{1}^{1}\left(x_{-1}^{*}\right)+\sum_{n=2}^{N} x_{n}^{*} \pi_{1, n}^{1}\left(x_{-(1, n)}^{*}\right)=\prod_{n=2}^{N}\left(1-x_{n}^{*}\right),
$$

as desired. 